\title{
Hennebert's Sign in Superior Semicircular Canal Dehiscence Syndrome: A Video Case Report
}

Andrew G. Shuman, MD; Syed S. Rizvi, MD; Chantale W. Pirouet, AuD; Katherine D. Heidenreich, MD

Superior semicircular canal dehiscence (SSCD) syndrome has been called the great otologic mimicker because its presentation overlaps with otosclerosis, Meniere's disease, perilymphatic fistula, and patulous eustachian tube. A valuable examination finding that can help distinguish SSCD syndrome from other pathologic conditions is the presence of Hennebert's sign, in which pressure changes in the external auditory canal evoke stereotyped eye movements that align in the plane of the dehiscent semicircular canal. This video case report demonstrates Hennebert's sign associated with SSCD syndrome and discusses its pathophysiological basis.

Key Words: Superior semicircular canal dehiscence, fistula test, Hennebert's sign, nystagmus.

Laryngoscope, 122:412-414, 2012

\section{CASE REPORT}

A 46-year-old man presented to a tertiary care otology clinic with a 3-year history of gradually progressive left hearing loss associated with ipsilateral autophony and pulsatile tinnitus. He reported occasional disequilibrium when exposed to loud sounds. He denied antecedent head trauma. His past medical history was significant for human immunodeficiency virus, with a normal CD4 count and no history of syphilis or acquired immune deficiency syndrome-defining illnesses.

On physical examination, the Weber test lateralized to the left ear. The left Rinne test showed bone conduction heard better than air conduction at $512 \mathrm{~Hz}$. The right Rinne test showed air conduction heard better than bone conduction at $512 \mathrm{~Hz}$. The tympanic membrane was intact, translucent, and mobile bilaterally. With video-oculography goggles on to suppress visual fixation, pneumatic otoscopy was performed using a Siegle speculum. In the left ear, positive pressure evoked a conjugate vertical-torsional ocular deviation where the

From the Division of Head and Neck Surgery (A.G.S.), Memorial Sloan-Kettering Cancer Center, New York, New York, U.S.A; Division of Otology and Neurotology, Department of Otolaryngology-Head and Neck Surgery (s.S.R., K.D.H.), University of Michigan Health System, Ann Arbor, Michigan, U.S.A., and Hearing Wellness Centre (c.w.P.), Windsor, Ontario, Canada.

Editor's Note: This Manuscript was accepted for publication October 3, 2011.

The authors have no funding, financial relationships, or conflicts of interest to disclose.

Send correspondence to Katherine D. Heidenreich, MD, Division of Otology and Neurotology, Department of Otolaryngology-Head and Neck Surgery, 1904 Taubman Center, 1500 E. Medical Center Drive, Ann Arbor, MI 48109. E-mail: kheidenr@med.umich.edu

DOI: 10.1002/lary.22413 eyes rotated up and away from the left ear. This reversed with negative pressure (Fig. 1 and online video). No nystagmus could be triggered with pressure changes in the right external auditory canal. Exposing either ear to loud sound from a Bárány noisemaker did not cause nystagmus. The patient was not asked to perform a Valsalva maneuver. The rest of a comprehensive neuro-otologic examination was normal.

Audiometry demonstrated normal hearing in the right ear. The left ear had mild low frequency conductive hearing loss with excellent word discrimination score. The left ear had a low vestibular evoked myogenic potential (VEMP) threshold of $65 \mathrm{~dB}$. The VEMP threshold was normal in the right ear. Electrocochleography (ECoG) revealed an elevated summating potential (SP) to action potential (AP) ratio of 0.58 in the left ear and a normal SP/AP value in the right ear. A temporal bone computed tomography (CT) scan reformatted in the Poschl and Stenver planes demonstrated dehiscent bone over the left superior semicircular canal (Fig. 2).

The patient was diagnosed with left SSCD syndrome and was offered surgical plugging of the canal via a middle fossa approach, which he declined.

\section{DISCUSSION}

Hennebert's sign can be broadly defined as eye movements evoked by pressure changes in the external auditory canal. ${ }^{1}$ The sign was named after Camille Hennebert (1867-1954), a Belgian otolaryngologist who described this finding among subjects with presumed congenital syphilis. ${ }^{2,3}$ He observed a conjugate horizontal nystagmus in these subjects, all of whom had an intact 


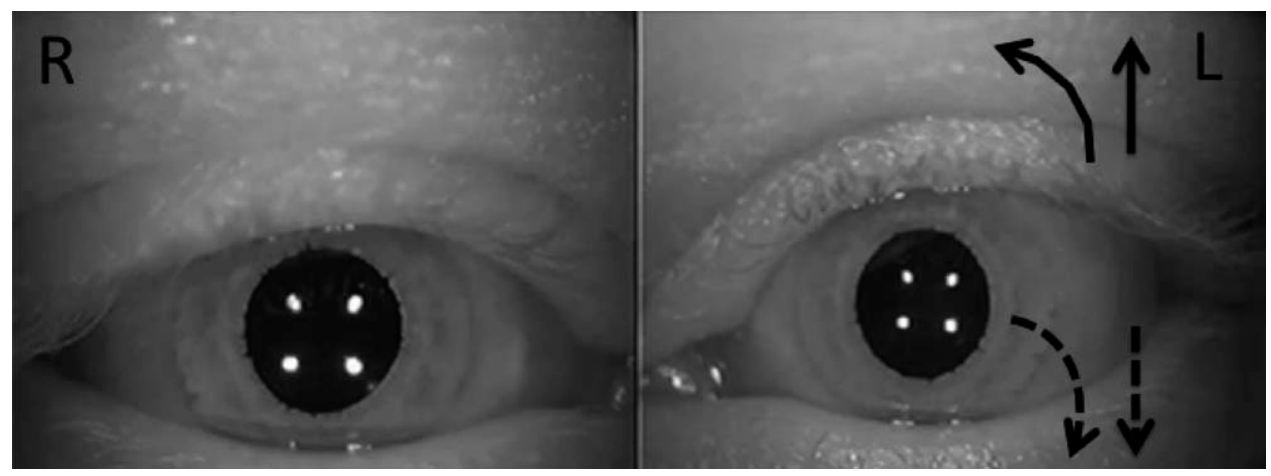

Fig. 1. Hennebert's sign associated with left superior semicircular canal dehiscence syndrome as seen under video-oculography goggles. The video with audio is available online. Positive pressure in the left ear causes a conjugate vertical-torsional ocular deviation where the eyes rotate up and away from the left ear (solid arrows). This reverses with negative pressure (dotted arrows). These eye movements align in the plane of the left superior semicircular canal. $R=$ right; $L=$ left.

tympanic membrane with no apparent middle ear disease. Up to this point, eye movements caused by pressure changes in the ear canal were reported almost exclusively in those with labyrinthine fistula secondary to chronic suppurative otitis media.

Hennebert's sign has been described in various pathologic conditions of the ear. When present in SSCD syndrome, the evoked eye movement aligns in the plane of the superior semicircular canal, providing an important clue regarding the site of pathology. The pathophysiologic basis for this particular eye movement is the creation of a third mobile window in the inner ear from the bony dehiscence. This permits abnormal pressure transmission through the labyrinth, with preferential shunting of endolymph along the affected canal. Positive pressure in the external canal causes a forceful inward movement of the tympanic membrane that is transmitted to the stapedial footplate. This leads to ampullofugal endolymph flow in the superior semicircular canal that excites afferent nerves. The brain perceives excitation of the left superior semicircular canal as a head movement down and rolled to the left; the vestibular ocular reflex then compensates by generating a slow phase eye movement that is upward and toward the right. Negative pressure in the external auditory is believed to cause prolapse of dura into the dehiscence, which leads to ampullopetal endolymph flow in the superior semicircular canal. ${ }^{4}$ This inhibits vestibular afferents in the superior semicircular canal and manifests as a slow, downward, torsional eye movement where the superior pole of the eye rotates toward the affected ear.

The exact prevalence of Hennebert's sign among the SSCD syndrome population remains unknown. Absence of this sign does not exclude SSCD syndrome. Some patients may develop eye movements in response to other stimuli such as loud sound (Tullio phenomenon) or a Valsalva maneuver. Loud sound or Valsalva against pinched nostrils causes ampullofugal endolymph flow in the superior canal which excites canal afferents. The patient exhibits a sustained nystagmus where the slow phase is up and rotates away from the affected ear. Valsalva against a closed glottis causes increased intracranial pressure, which exerts a downward force at the site of dehiscence. This results in ampullopetal endolymph flow in the canal, and the patient develops an inhibitory nystagmus with a slow phase that moves down and rotates toward the affected ear. Considerable variability exists in the clinical presentation of SSCD syndrome for reasons that remain unknown. A formal diagnosis of SSCD syndrome requires radiographic evidence of absent bone over the semicircular canal on
Fig. 2. Left superior semicircular canal dehiscence as seen on computed tomography scan of the temporal bone. (A) Poschl plane. (B) Stenver plane. The arrow points to the region of dehiscence.
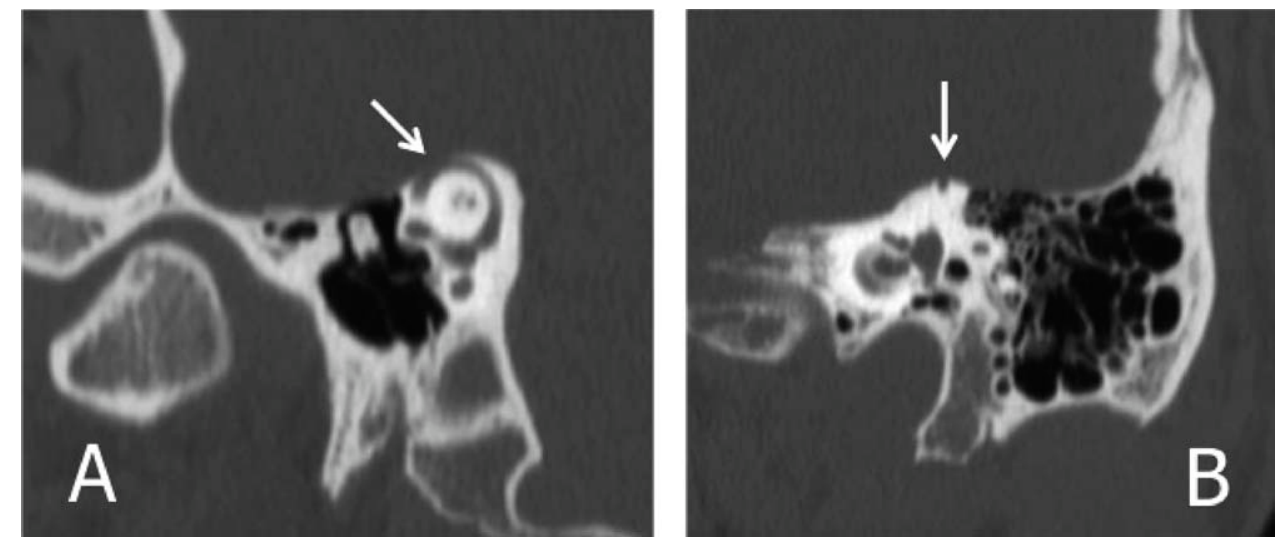
multiplanar CT of the temporal bones in addition to any one of the following physiologic signs: decreased VEMP thresholds, conductive hearing loss of at least $10 \mathrm{~dB}$ at one frequency between 250 to $4,000 \mathrm{~Hz}$, elevated SP/AP ratio on ECoG, or pressure or tone-evoked eye movements. ${ }^{5-7}$

\section{CONCLUSION}

Hennebert's sign can aid in the diagnosis of SSCD syndrome based on a detailed understanding of its pathophysiology. Patients with SSCD syndrome who exhibit Hennebert's sign develop eye movements that align in the plane of the affected semicircular canal in response to pressure changes in the ipsilateral external auditory canal. We have provided videographic documentation of this phenomenon. Despite the increased reliance on sophisticated neurophysiologic testing and imaging modalities in the evaluation of otologic disease, it is important to recognize that a clinical sign described over 100 years ago remains relevant and useful today.

\section{BIBLIOGRAPHY}

1. Chien WW, Carey JP, Minor LB. Canal dehiscence. Curr Opin Neurol 2011; 24:25-31.

2. Hennebert C. Reflexe oto-oculo-moteur. Internationales Zentralblatt fur Ohrenheilkunde und Rhino-Laryngologie 1905;3:405.

3. Hennebert C. Reactions vestibulaires dans les labyrinthites heredo-syphilitiques. Archives internationals de laryngology, otologie-rhinologie, brocho-oesophagoscopie 1909;28:93-96.

4. Hullar TE, Zee DS, Minor LB. Evaluation of the dizzy patient. In: Flint PW Haughey BH, Lund VJ, et al., eds. Cummings Otolaryngology-Head and Neck Surgery. 5th ed. Philadelphia, PA: Mosby Elsevier; 2010:23052327.

5. Minor LB. Clinical manifestations of superior semicircular canal dehiscence. Laryngoscope 2005;115:1717-1727.

6. Crane BT, Minor LB, Carey JP. Three-dimensional computed tomography of superior canal dehiscence syndrome. Otol Neurotol 2008;29:699-705.

7. Arts HA, Adams ME, Telian SA, El-Kashlan HK, Kileny PR. Reversible electrocochleographic abnormalities in superior canal dehiscence. Otol Neurotol 2008;30:79-86. 